\title{
EIGENVALUES OF KINEMATICAL CONSERVATION LAWS (KCL) BASED 3-D WEAKLY NONLINEAR RAY THEORY (WNLRT)
}

\author{
K. R. ARUN AND PHOOLAN PRASAD
}

\begin{abstract}
Kinematical conservation laws (KCL) is a system of conservation laws governing the evolution of a curve in a plane or a surface in space, even if the curve or the surface has singularities on it. In our recent publication [1] we have developed a mathematical theory to study the successive positions and geometry of a 3-D weakly nonlinear wavefront by adding an energy transport equation to KCL. The $7 \times 7$ system of equations of this KCL based 3 -D weakly nonlinear ray theory (WNLRT) is quite complex and explicit expressions for its two nonzero eigenvalues could not be obtained in [1]. In this short note, we use two different methods: (i) the equivalence of KCL and ray equations and (ii) the transformation of surface coordinates, to derive the same exact expressions for these eigenvalues. The explicit expressions for nonzero eigenvalues are important also for checking stability of any numerical scheme to solve 3-D WNLRT.
\end{abstract}

\section{INTRODUCTION}

In [1] we have developed a method to study the propagation of a surface $\Omega_{t}$ in three-dimensional space $\mathbb{R}^{3}$. The paper contains two systems of equations for an isotropic evolution of $\Omega_{t}$. The first system, called the ray equations, is for the evolution of a smooth $\Omega_{t}$ and consists of equations ${ }^{1}$

$$
\begin{aligned}
& \frac{\mathrm{d} \mathbf{x}}{\mathrm{d} t}=m \mathbf{n},|\mathbf{n}|=1, \\
& \frac{\mathrm{d} \mathbf{n}}{\mathrm{d} t}=-\mathbf{L} m:=-(\nabla-\mathbf{n}\langle\mathbf{n}, \nabla\rangle) m .
\end{aligned}
$$

The second system, called 3-D KCL, consists of six conservation laws

$$
\begin{aligned}
& \left(g_{1} \mathbf{u}\right)_{t}-(m \mathbf{n})_{\xi_{1}}=0 \\
& \left(g_{2} \mathbf{v}\right)_{t}-(m \mathbf{n})_{\xi_{2}}=0 .
\end{aligned}
$$

The KCL (1.3)-(1.4) is to be supplemented by an additional condition $\left(g_{2} \mathbf{v}\right)_{\xi_{1}}-\left(g_{1} \mathbf{u}\right)_{\xi_{2}}=0$ to be satisfied at time $t=0$. Unlike the ray equations, the KCL remains valid also when $\Omega_{t}$ develops singularities.

As long as $\Omega_{t}$ remains smooth, the systems (1.1)-(1.2) and (1.3)-(1.4) are equivalent, we refer the reader to [1] for a proof of this basic result.

It is to be pointed out that both the systems (1.1)-(1.2) and (1.3)-(1.4) are under-determined. Hence, suitable closure relations are to be supplied to both to get a completely determined system of equations. In [1] we have derived a closure relation by considering the energy propagation along the rays of a weakly nonlinear ray theory (WNLRT) under high frequency approximation. For a

Date: May 26, 2009.

2000 Mathematics Subject Classification. Primary 35L65, 35L67; Secondary 35L80.

Key words and phrases. kinematical conservation laws, ray theory, weakly nonlinear wave front, polytropic gas.

${ }^{1}$ For definition of various quantities and notations, see the reference [1], particularly Appendix B. 
nonlinear wave front running into a polytropic gas at rest and in uniform state, the energy transport equation written in the form (7.4) of [1] is

$$
\frac{\partial m}{\partial t}=\frac{m-1}{2 n_{3}}\left(n_{1} \frac{\partial}{\partial x_{3}}-n_{3} \frac{\partial}{\partial x_{1}}\right) n_{1}-\frac{m-1}{2 n_{3}}\left(n_{3} \frac{\partial}{\partial x_{2}}-n_{2} \frac{\partial}{\partial x_{3}}\right) n_{2} .
$$

A conservation form of the equation (1.5) is the equation (6.7) of [1] which is

$$
\left\{(m-1)^{2} e^{2(m-1)} g_{1} g_{2} \sin \chi\right\}_{t}=0 .
$$

We add the differential form the energy transport equation, namely (1.5) to the ray equations (1.1)-(1.2). Henceforth, we shall designate the resulting system of equations as ray equations based WNLRT. Similarly, we will refer the KCL (1.3)-(1.4) along with the energy equation (1.6) in conservation form as $K C L$ based WNLRT.

Since the two formulations of WNLRT are equivalent for smooth solutions, their nonzero eigenvalues must be same, see [1]-Section 8.4. The aim of the present work is to determine these eigenvalues of the KCL based WNLRT from the much simpler system of ray equations based WNLRT.

\section{Calculation of the Nonzero Eigenvalues}

For the determination of eigenvalues, we do not need the first part of the ray equations, i.e., equation (1.1) but only the equation (1.2) for $\mathbf{n}$. However, since $|\mathbf{n}|=1$, the vector equation (1.2) contains only two independent scalar equations. The two evolution equations for $n_{1}$ and $n_{2}$ are equations (5.9)-(5.10) of [1] which we reproduce below.

$$
\begin{aligned}
& \frac{\partial n_{1}}{\partial t}=-\frac{1}{\sin ^{2} \chi}\left\{\left(u_{1}-v_{1} \cos \chi\right) \frac{\partial m}{g_{1} \partial \xi_{1}}+\left(v_{1}-u_{1} \cos \chi\right) \frac{\partial m}{g_{2} \partial \xi_{2}}\right\}, \\
& \frac{\partial n_{2}}{\partial t}=-\frac{1}{\sin ^{2} \chi}\left\{\left(u_{2}-v_{2} \cos \chi\right) \frac{\partial m}{g_{1} \partial \xi_{1}}+\left(v_{2}-u_{2} \cos \chi\right) \frac{\partial m}{g_{2} \partial \xi_{2}}\right\} .
\end{aligned}
$$

Using the mapping between $\left(x_{1}, x_{2}, x_{3}\right)$-space and $\left(\xi_{1}, \xi_{2}, t\right)$-space, the expressions for the partial derivatives $\frac{\partial}{\partial x_{1}}, \frac{\partial}{\partial x_{2}}$ and $\frac{\partial}{\partial x_{3}}$ can be derived in terms of $\frac{\partial}{\partial t}, \frac{\partial}{\partial \xi_{1}}$ and $\frac{\partial}{\partial \xi_{2}}$, see (5.6) of [1]. After the transformation of independent variables to $\left(\xi_{1}, \xi_{2}, t\right)$, the energy transport equation (1.5) becomes

$$
\frac{\partial m}{\partial t}=\frac{m-1}{2 n_{3} \sin \chi}\left(v_{2} \frac{\partial n_{1}}{g_{1} \partial \xi_{1}}+v_{1} \frac{\partial n_{2}}{g_{1} \partial \xi_{1}}-u_{2} \frac{\partial n_{1}}{g_{2} \partial \xi_{2}}-u_{1} \frac{\partial n_{2}}{g_{2} \partial \xi_{2}}\right) .
$$

It is to be remarked that though the variables $g_{1}$ and $g_{2}$ do appear in equations (2.1)-(2.3), no derivatives of $g_{1}$ and $g_{2}$ are present. Hence, for the calculation of the nonzero eigenvalues, we need not consider the evolution equations for $g_{1}$ and $g_{2}$ given by the equations (6.10)-(6.11) of [1]. We can easily write down the characteristic equation of (2.1)-(2.3) and solve for the eigenvalues, which turn out to be

$$
\lambda_{1}, \lambda_{2}=\left(-\lambda_{1}\right), \lambda_{3}=0,
$$

where

$$
\lambda_{1}=\left\{\frac{m-1}{2 \sin ^{2} \chi}\left(\frac{e_{1}^{2}}{g_{1}^{2}}-\frac{2 e_{1} e_{2}}{g_{1} g_{2}} \cos \chi+\frac{e_{2}^{2}}{g_{2}^{2}}\right)\right\}^{1 / 2} .
$$

The explicit expressions for $\lambda_{1}$ and $\lambda_{2}$ in (2.4)-(2.5) are simple and elegant. They reduce to $\nu_{1}$ and $\nu_{2}$ in equation (8.2) of [1] when $\mathbf{u}$ and $\mathbf{v}$ are orthogonal. The analytical expressions for nonzero eigenvalues of KCL are essential to determine a suitable CFL stability condition in the numerical integration of conservation laws of KCL based WNLRT, see [2] for more details. 


\section{Calculation of nonzero eigenvalues by transformation of surface coordinates AT A POINT.}

In this section we shall use the method of transformation of coordinates explained in section 8.3 of [1] to deduce the expression (2.5). Given the surface coordinates $\left(\xi_{1}, \xi_{2}\right)$ at a point $P_{0}$ on $\Omega_{t}$ with unit vectors $(\mathbf{u}, \mathbf{v})$, we choose an orthogonal coordinates at $P_{0}$ with unit vectors $\left(\mathbf{u}^{\prime}, \mathbf{v}^{\prime}\right)$ as follows

$$
\mathbf{u}^{\prime}=\mathbf{u}, \quad \mathbf{v}^{\prime}=\gamma_{2} \mathbf{u}+\delta_{2} \mathbf{v},
$$

where $\left\langle\mathbf{u}^{\prime}, \mathbf{v}^{\prime}\right\rangle=0$ and $\left|\mathbf{v}^{\prime}\right|=1$ so that

$$
\gamma_{2}=-\cot \chi, \quad \delta_{2}=\operatorname{cosec} \chi
$$

The sign of $\gamma_{2}$ and $\delta_{2}$ are so chosen that vectors $\mathbf{u}^{\prime}, \mathbf{v}^{\prime}$ and $\mathbf{n}^{\prime}$ form a right handed system, similar to the three vectors $\mathbf{u}, \mathbf{v}$ and $\mathbf{n}$. Substituting $\gamma_{1}=1, \delta_{1}=0$ and the above values of $\gamma_{2}$ and $\delta_{2}$ in the expression (8.15) of [1] we get the same expression (2.5) for $\lambda_{1}$.

\section{Concluding Remarks}

Though we could not derive the expression for $\lambda_{1}$ by solving the characteristic equation $\operatorname{det}(-\lambda A+$ $\left.e_{1} B^{(1)}+e_{2} B^{(2)}\right)=0$ of the system (6.12) of [1], we have obtained its explicit expression (2.5) by two different methods. This leaves no doubt about the correctness of the expression in (2.5). However, just as a check, we have numerically evaluated the expression (2.5) and numerically solved the eigenvalue problem of the KCL based 3-D WNLRT with $g_{1}=g_{2}=1$ and various values of $\mathbf{u}, \mathbf{v}$ and $m$. The numerical values of $\lambda_{1}$ agree in all cases, see also section (8.2) of [1].

\section{ACKNOWLEDGEMENT}

Work of KRA is supported by the Council of Scientific \& Industrial Research (CSIR) under the grant-09/079(2084)/2006-EMR-1. PP's research is supported by the Department of Atomic Energy, Government of India under Raja Ramanna Fellowship Scheme. The authors gratefully acknowledge their respective grants. Department of Mathematics of IISc is partially funded by UGC under SAP.

\section{REFERENCES}

[1] K. R. Arun and P. Prasad. 3-D kinematical conservation laws (KCL): evolution of a surface in $\mathbb{R}^{3}$-in particular propagation of a nonlinear wavefront. Wave Motion (2009), doi:10.1016/j.wavemoti.2009.03.004.

[2] K. R. Arun, M. Lukáčová-Medvidová, Phoolan Prasad, and S. V. Raghurama Rao. An application of 3-D kinematical conservation laws: propagation of a three dimensional wavefront. Preprint, Department of Mathematics, Indian Institute of Science, 2008.

(K. R. Arun and Phoolan Prasad) Department of Mathematics, Indian Institute of Science, BangaLORE, INDIA-560012.

E-mail address: prasad@math.iisc.ernet.in

URL: http://math.iisc.ernet.in/ prasad/ 